\section{Centros de Atenção Psicossocial Infantojuvenil no Brasil: distribuição geográfica e perfil dos usuários}

\author{
Psychosocial Care Centers for Children and \\ Adolescents in Brazil: geographic \\ distribution and user profile
}

\author{
Centros de Atención Psicosocial Infanto-Juvenil en \\ Brasil: distribución geográfica \\ y perfil de sus usuarios
}

\author{
1 Instituto de Saúde Coletiva, \\ Universidade Federal da \\ Bahia, Salvador, Brasil. \\ Correspondência \\ G. Y. C. Garcia \\ Instituto de Saúde Coletiva, \\ Universidade Federal da \\ Bahia. \\ Rua Basílio da Gama, \\ Campus Universitário do \\ Canela, Salvador, $B A$ \\ 40110-040, Brasil. \\ greyyuliet@hotmail.com
}

\begin{abstract}
Few Brazilian studies have addressed the use of mental health services for children and adolescents. This study aimed to characterize the national distribution of Psychosocial Care Centers for Children and Adolescents (CAPSi) and describe the patient profile in this age group between 2008 and 2012. An ecological study was carried out, using records from the Authorizations for High-Complexity Procedures (APAC) system and the Brazilian National Registry of Healthcare Organizations (CNES). Socio-demographics and disease profile were analyzed. In 2014, 208 CAPSi were recorded in the CNES, distributed across 23 of Brazil's 27 states. Treatments included predominantly behavioral disorders (29.7\%), developmental disorders (23.6\%), and mental retardation (12.5\%). CAPSi are insufficient and unequally distributed. The disease profile suggests the need for linkage between specialized mental health services and primary care, in addition to the inclusion of inter-sector work.
\end{abstract}

Mental Health Services; Child; Adolescent; Mental Health
Grey Yuliet Ceballos Garcia 1

Darci Neves Santos 1

Daiane Borges Machado 1

\section{Resumo}

Poucos estudos brasileiros abordam a utilização de serviços de saúde mental para população infanto-juvenil. Neste trabalho, objetivou-se caracterizar a distribuição nacional dos Centros de Atenção Psicossocial Infantojuvenis (CAPSi) e descrever o perfil nosológico dos atendimentos infantojuvenis entre 2008 e 2012. Realizou-se estudo ecológico, utilizando registros do sistema de Autorizações de Pagamento de Serviços de Alta Complexidade (APAC) e do Cadastro Nacional de Estabelecimentos de Saúde (CNES). Aspectos sociodemográficos e perfil nosológico foram analisados. Em 2014, 208 CAPSi estavam registrados no CNES, distribuíam-se em 23 das 27 unidades da federação brasileira. Nos atendimentos, predominaram transtornos de comportamento (29,7\%), transtornos de desenvolvimento (23,6\%) e retardo mental (12,5\%). Os CAPSi são insuficientes e desigualmente distribuídos. O perfil nosológico sugere necessidade de articulação entre serviços especializados de saúde mental e atenção básica, além de inclusão do trabalho intersetorial.

Serviços de Saúde Mental; Criança; Adolescente; Saúde Mental 


\section{Introdução}

As prevalências brasileiras de problemas de saúde mental em crianças e adolescentes variam entre $7 \%$ e $24,6 \% 1$, com demanda entre $4 \%$ e $7,3 \%$ para intervenção clínica 1 . Contudo, poucos estudos abordam a utilização de serviços de saúde mental nesse grupo populacional 2,3.

A lacuna entre necessidade de tratamento e acesso aos serviços de saúde mental é mundialmente reconhecida 4 .

Cabe aos Centros de Atenção Psicossocial Infantojuvenil (CAPSi), instituídos pela Lei no 10.216/2001 5, a chamada Lei da Reforma Psiquiátrica, acolher crianças e adolescentes com transtornos mentais graves.

Trata-se de um estudo pioneiro no uso de dados procedentes das Autorizações de Pagamento de Serviços de Alta Complexidade (APAC), em associação com informações do Cadastro Nacional de Estabelecimentos de Saúde (CNES), visando caracterizar a distribuição nacional dos CAPSi e descrever o perfil nosológico dos atendimentos infantojuvenis.

\section{Métodos}

Estudo descritivo, ecológico, utilizando dados das APAC entre 2008 e 2012 e do CNES entre 2001 e 2014. Adotou-se como unidade de análise o CAPSi que registrou atendimentos a menores de 19 anos no primeiro período assinalado.

Sexo e idade foram as variáveis demográficas analisadas. Determinou-se o perfil nosológico conforme diagnósticos do grupo V (F00-F99) da Classificação Internacional de Doenças - 10ạ revisão (CID-10).

Para obter as taxas de atendimento, dividiuse o total de atendimentos do CAPSi pela população abaixo de 19 anos por estado e região, para cada ano entre 2008 e 2012. Utilizou-se o parâmetro populacional de 150 mil habitantes, conforme Portaria no 3.088/2011 para a implantação de CAPSi 6 . Todavia, essa portaria, atualizada em 2013, instituiu o parâmetro de 70 mil habitantes para implantação de CAPSi. Optou-se pelo parâmetro antigo porque o estudo utilizou dados do CNES entre 2001 e 2014.

Os dados foram processados com os softwares TabWin (Departamento de Informática do SUS. http://www2.datasus.gov.br/DATASUS/in dex.php?area=060805) e Stata, versão 12 (StataCorp LP, College Station, Estados Unidos). A pesquisa não exigiu submissão ao Comitê de Ética, por abordar dados públicos de livre acesso.

\section{Resultados}

\section{Descrição da rede nacional de CAPSi}

Pelo CNES, 208 CAPSi funcionavam em abril de 2014, 32 dos quais sem registro de habilitação, e $98,1 \%$ sob gestão municipal. Distribuíam-se em 23 das 27 unidades federativas (UF) brasileiras, estando ausentes no Acre, Roraima, Tocantins e Distrito Federal.

Dentre 187 municípios com mais de 150 mil habitantes, apenas $60,4 \%$ possuíam CAPSi, havendo distribuição heterogênea nos estados. O percentual de municípios com pelo menos um CAPSi oscilou entre $60 \%$ e $75 \%$ segundo regiões, exceto a Região Norte com 38,5\%. Na Região Sul, 74,2\% dos municípios possuíam esse serviço. Apesar de o Estado de São Paulo possuir maior número de CAPSi, apenas $51 \%$ das cidades habilitadas pelo critério populacional estavam contempladas. Até abril de 2014, os CAPSi representavam 7,8\% do total de 2.128 Centros de Atendimento Psicossocial (CAPS) brasileiros, com 52,9\% localizados no Sudeste.

Se cada CAPSi atendia mensalmente 155 pacientes, havendo 208 deles em 2014, teriam sido atendidos 32.240 usuários/mês. Considerando que entre $4 \%$ e $7,3 \%$ da população infantojuvenil requer intervenção clínica, essa capacidade instalada somente atenderia entre $0,72 \%$ e 1,32\% da demanda.

As taxas de atendimento dos CAPSi no Brasil variaram de 185 atendimentos por $100 \mathrm{mil}$ habitantes < 19 anos, em 2008, a 325,6/100.000 em 2012, demonstrando tendência crescente no período estudado. Variações menores ocorreram na Região Norte, entre 0,8/100.000 em 2008 e $53,1 / 100.000$ em 2012. No Sudeste, as taxas foram similares à média nacional, enquanto no Nordeste e Centro-oeste apresentaram valores abaixo da média. No Sul, houve variação expressiva, entre 377,8/100.000 em 2008 e 581,5/100.000 em 2012 (Figura 1).

\section{Perfil nosológico dos atendimentos}

Foram analisados 837.259 registros de APAC, com $65,8 \%$ dos atendimentos concentrados em três grupos diagnósticos: $29,7 \%$ para transtornos do comportamento e transtornos que aparecem habitualmente durante a infância ou a adolescência (F90-F98), 23,6\% para transtornos do desenvolvimento psicológico (F80-F89); e 12,5\% para retardo mental (F70-F79).

Esse perfil nosológico variou conforme regiões, sendo mais frequente no Norte e Nordeste o diagnóstico de retardo mental. Os diagnósticos de transtornos do comportamento e emocionais 
Taxa de atendimento infantojuvenil em Centros de Atenção Psicossocial Infantojuvenis (CAPSi) vs. população < 19 anos, por regiões do Brasil, 2008-2012.

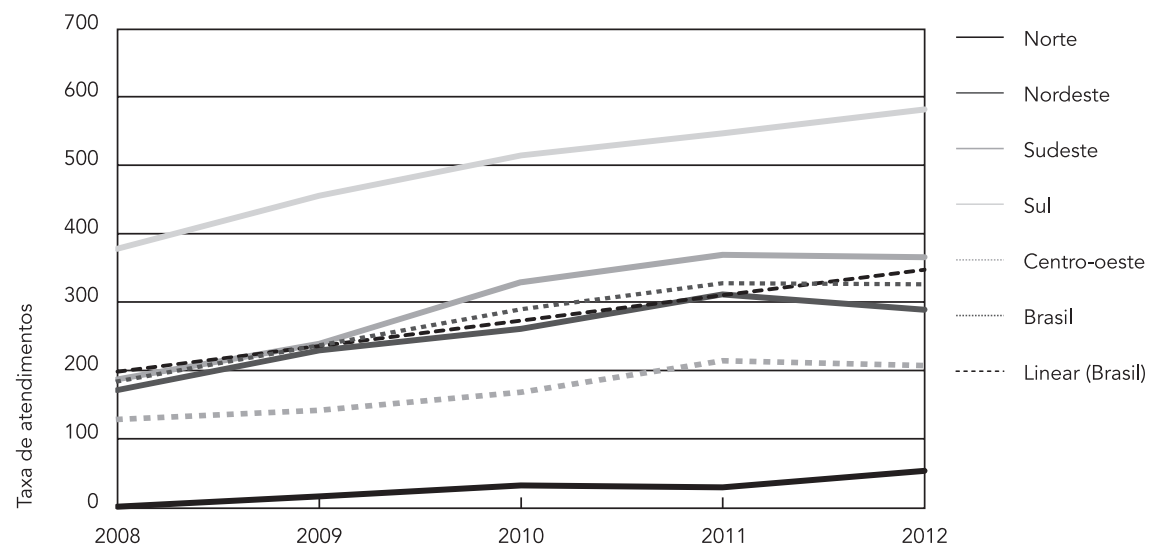

foram os mais frequentes em quatro regiões, sendo ultrapassados no Sudeste pelos de transtornos do desenvolvimento psicológico. Os transtornos do desenvolvimento psicológico alternaram sua segunda posição com transtornos de humor no Centro-oeste, e com retardo mental na Região Norte (Figura 2).

Entre diagnósticos menos frequentes, destacaram-se os transtornos neuróticos, transtornos relacionados com o stress e transtornos somatoformes com 10,4\% e os transtornos pelo uso de substâncias psicoativas com 1,8\% (dados não apresentados nas figuras).

\section{Discussão}

A rede atual de CAPSi é ainda insuficiente e desigualmente distribuída. Sendo um serviço público predominantemente municipal, pode-se atribuir tal situação à ausência de decisão política e de investimento para ampliação dos serviços, além da escassez de psiquiatras gerais e de especialistas em psiquiatria infantojuvenil. Esses profissionais situam-se em grandes centros urbanos, principalmente na Região Sudeste, onde se concentram $76 \%$ das residências em psiquiatria 7 .

A Organização Mundial da Saúde (OMS) tem enfatizado a insuficiência, no Brasil, de serviços infantojuvenis. Estimativas sugerem que 258 CAPSi seriam necessários para atender portado- res de transtornos do desenvolvimento menores de 20 anos no Estado de São Paulo 4 .

Os achados deste trabalho sugerem a necessidade de aumento da rede de CAPSi e melhoria na distribuição por regiões, contrariando avaliação, em 2012, da implementação nacional da rede de CAPS 8. O indicador de cobertura incluiu no numerador os três tipos de CAPS, obtendo-se uma cobertura de 0,74 CAPS/100.000 habitantes, considerada muito boa ${ }^{9}$. As limitações desse indicador foram reconhecidas pelo Ministério da Saúde, referindo necessidade de construir indicadores sensíveis à cobertura de CAPSi, embora seja desconhecida qualquer iniciativa até o momento.

\section{Perfil nosológico dos CAPSi}

O perfil nosológico foi similar àqueles anteriormente observados 3,10 , estando os transtornos de comportamento e do desenvolvimento entre as três primeiras causas de utilização dos serviços, porém registros por consumo de substâncias psicoativas não foram anteriormente encontrados.

Os transtornos comportamentais infantojuvenis requerem condução adequada, pois tendem a persistir no curso de vida, associando-se à delinquência, comportamento antissocial, problemas conjugais e saúde física deficitária. Cabe refletir sobre espaços apropriados para acolhimento desses jovens, diante da evidência de que 
Figura 2

Diagnósticos mais frequentes nos Centros de Atenção Psicossocial Infantojuvenis (CAPSi) por regiões do Brasil, $2008-2012$.

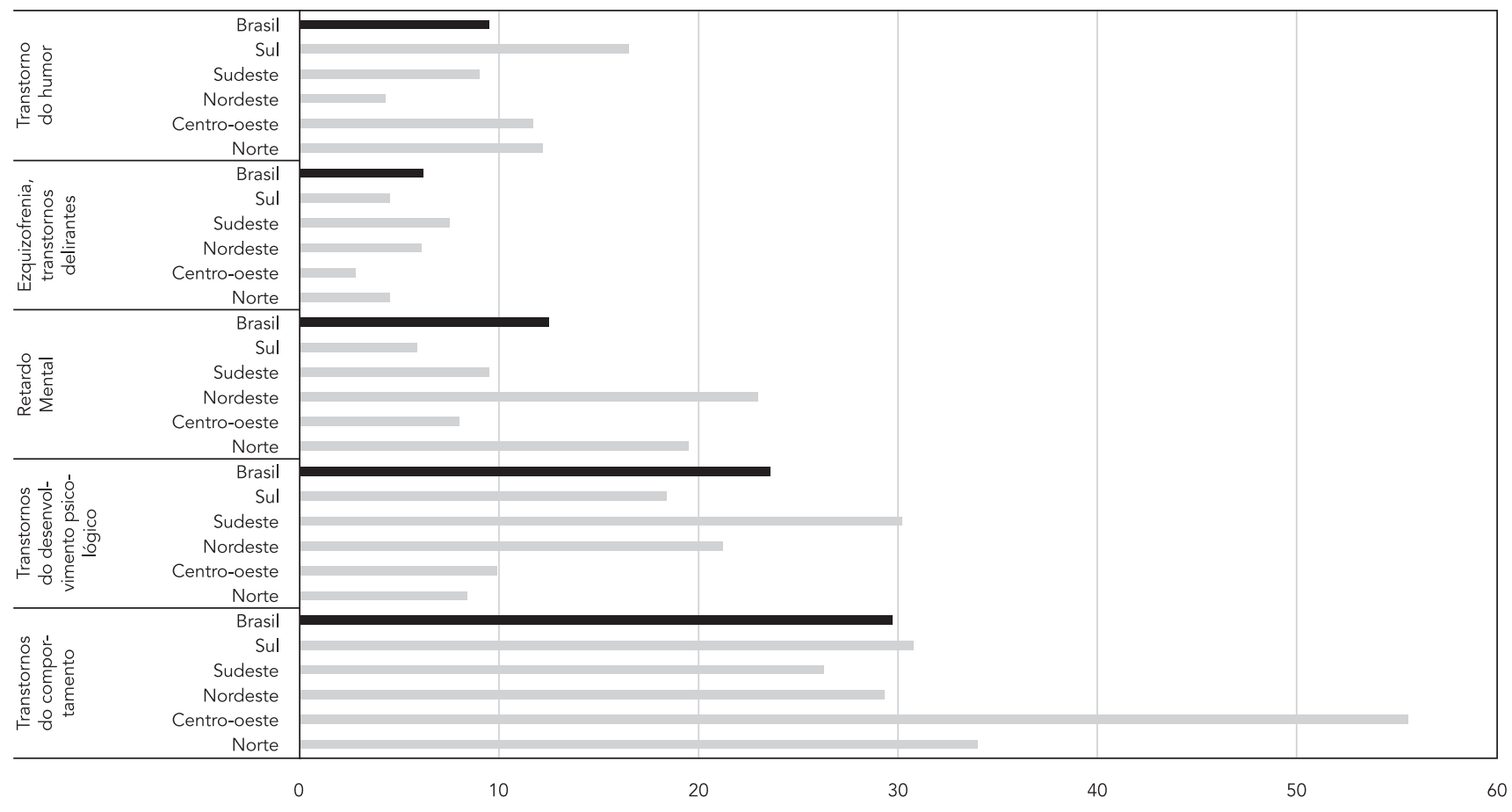

cuidados de saúde mental na atenção básica se associam com redução de estigma e melhor engajamento do paciente e sua família ${ }^{4}$.

Cabe, ainda, articular serviços especializados de saúde mental infantojuvenil com atenção básica, pela carência de unidades e de recursos humanos especializados. Treinamento e suporte contínuo das equipes de saúde mental para profissionais da atenção básica favoreceriam a responsabilidade compartilhada. As atividades dos Núcleos de Apoio à Saúde da Família (NASF) buscam contemplar esses aspectos 11. O matriciamento tem sido bem avaliado quando as equipes conseguem adequada interlocução, estabelecendo corresponsabilidade no tratamento dos usuários 12,13.

A presença do retardo mental ao lado dos transtornos comportamentais revela a importância da articulação intersetorial, sobretudo com o setor educação, melhorando a gestão e integrando esforços na perspectiva longitudinal do desenvolvimento de crianças e adolescentes 9 .

\section{Limitações do estudo}

Em razão do uso de dados secundários, apontam-se algumas limitações. A ausência de identificação nominal poderia comprometer inferências populacionais, porque vários atendimentos a uma mesma pessoa poderiam ter ocorrido; no entanto, esses resultados estão alinhados com achados de estudos que empregaram dados primários e individuados.

Apesar da imprecisão no cálculo das taxas de atendimento, estima-se que aproximadamente $2 \%$ da demanda nacional estariam sendo atendidas.

Os resultados são também influenciados pelo período de disponibilidade dos dados e pelo não preenchimento de determinadas variáveis. O CNES, enquanto fonte exclusiva da distribuição nacional dos CAPSi, gera lacunas porque sua atualização depende dos gestores locais, que nem sempre o fazem em tempo hábil. Portanto, as taxas de atendimento e a distribuição dos CAPSi devem ser cuidadosamente interpretadas. 


\section{Conclusão}

Embora existam políticas formuladas e avanços na implantação de serviços de base comunitária para atenção em saúde mental, o cuidado de crianças e adolescentes com problemas mentais continua sendo um desafio, no Brasil, pela escassez e distribuição desigual desses serviços entre UFs e regiões.

O perfil nosológico obtido pelos atendimentos realizados nos CAPSi demonstra elevados percentuais de transtornos de comportamento e de retardo mental, evidenciando a importância da integração da rede especializada com a atenção básica e da articulação intersetorial no território.

Finalmente, ressaltamos o conhecido potencial dos sistemas brasileiros de informação para produzir conhecimento sobre a utilização dos serviços de saúde com rapidez e baixo custo.

\section{Resumen}

Pocos estudios brasileños abordan la utilización de servicios de salud mental para la población infantojuvenil. En este trabajo se tuvo por objetivo caracterizar la distribución nacional de los Centros de Atención Psicosocial Infanto-Juvenil (CAPSi), y describir el perfil nosológico de las consultas infanto-juveniles entre 2008 y 2012. Fue realizado un estudio ecológico, utilizando registros del sistema de Autorizaciones de Procedimientos de Alta Complejidad (APAC) y del Catastro Nacional de Establecimientos de Salud (CNES). Se analizaron aspectos sociodemográficos y perfil nosológico. En 2014, 208 CAPSi estaban registrados en el CNES, se distribuían en 23 de los 27 estados brasileños. En las consultas, predominaron trastornos de comportamiento (29,7\%), trastornos de desarrollo $(23,6 \%)$ y retraso mental (12,5\%). Los CAPSi son insuficientes y están desigualmente distribuidos. El perfil nosológico sugiere una necesidad de articulación entre servicios especializados de salud mental y atención básica, además de la inclusión del trabajo intersectorial.

Servicios de Salud Mental; Niño; Adolescente Salud Mental

\section{Colaboradores}

G. Y. C. Garcia participou da concepção, planejamento, análise e interpretação dos dados; elaboração do manuscrito; e aprovação da versão final do manuscrito. D. N. Santos participou da concepção e planejamento, interpretação dos dados; contribuição significativa na elaboração de versões anteriores e revisão crítica do conteúdo; e aprovação da versão final do manuscrito. D. B. Machado contribuiu com sugestões metodológicas, análise e interpretação dos dados; e aprovação da versão final do manuscrito.

\section{Agradecimentos}

Ao Professor Eduardo Mota do Instituto de Saúde Coletiva da Universidade Federal da Bahia, por suas sugestões na análise dos dados. Ao CNPq pela bolsa de mestrado concedida a G. Y. C. Garcia. 


\section{Referências}

1. Paula CS, Duarte CS, Bordin IA. Prevalence of mental health problems in children and adolescents from the outskirts of Sao Paulo City: treatment needs and service capacity evaluation. Rev Bras Psiquiatr 2007; 29:11-7.

2. Hoffmann MCCL, Santos DN, Mota ELA. Caracterização dos usuários e dos serviços prestados por Centros de Atenção Psicossocial Infanto-Juvenil. Cad Saúde Pública 2008; 24:633-42.

3. Paula CS, Lauridsen-Ribeiro E, Wissow L, Bordin I, Eevans-Lacko S. How to improve the mental health care of children and adolescents in Brazil: actions needed in the public sector. Rev Bras Psiquiatr 2012; 34:334-41.

4. Kieling C, Baker-Henningham H, Belfer M, Conti G, Erten I, Omigbodun O, et al. Child and adolescent mental health worldwide: evidence for action. Lancet 2011; 378:1515-25.

5. Brasil. Lei no 10.216, de 6 de abril de 2001. Diário Oficial da União 2011; 9 abr.

6. Ministério da Saúde. Portaria no 3.088, de 23 de dezembro de 2011. Diário Oficial da União 2011; $30 \mathrm{dez}$.

7. Fernandes RLF, Citero VA, Nogueira-Martins LA, Mari JJ. Psychiatry career in Brazil: regional disparities, differences and similarities in an international context. Int Rev Psychiatry 2013; 25:486-92.

8. Ministério da Saúde. Caderno de diretrizes, objetivos, metas e indicadores: 2013-2015. Brasília: Ministério da Saúde; 2013.
9. Coordenação Geral de Saúde Mental, Álcool e Outras Drogas, Departamento de Ações Programáticas e Estratégicas, Secretaria de Atenção à Saúde, Ministério de Saúde. Saúde Mental em Dados 2012; ano VII, no 11. http:/ / www.saude.gov.br/bvs/ saudemental.

10. Delfini PSS, Dombi-Barbosa C, Fonseca LF, Tavares MC, Reis AOA. Perfil dos usuários de um centro de atenção psicossocial infantojuvenil da grande São Paulo, Brasil. Rev Bras Crescimento Desenvolv Hum 2009; 19:226-36.

11. Departamento de Atenção Básica, Secretaria de Atenção à Saúde, Ministério de Saúde. Diretrizes do NASF: Núcleo de Apoio à Saúde da Família. Brasília: Ministério da Saúde; 2010. (Cadernos de Atenção Básica, 27).

12. Onocko CR, Gama CA, Ferrer AL, Santos D, Stefanello S, Trape T, et al. Saúde mental na atenção primária à saúde: estudo avaliativo em uma grande cidade brasileira. Ciênc Saúde Coletiva 2011; 16:4643-52.

13. Delfini PSS, Reis AOA. Articulação entre serviços públicos de saúde nos cuidados voltados à saúde mental infantojuvenil. Cad Saúde Pública 2012; 28:357-66.

Recebido em 02/Abr/2015

Versão final reapresentada em 07/Set/2015

Aprovado em 15/Out/2015 